\title{
Differential payoff and locus of selectivity in a visual memory task
}

\author{
FRANK TOLKMITT $^{2}$ AND RICHARD E. CHRIST ${ }^{3}$ \\ KANSAS STATE UNIVERSITY
}

Two experiments were run to investigate the locus of selectivity in a visual memory task involving differential payoff for letters correctly recalled. Major variables consisted of value instructions given before or after the exposure of a display, the rutio of high to low value, and exposure duration. The results showed that $S$ s make more correct reports of higher value than of lesser value, whether value instructions are given before or after the display. Furthermore, overall performance and extent of selectivity increase with increasing exposure time, but more for the before than the after conditions of instruction. These results suggest that selectivity occurs both during perception and retention and during recall.

Recent studies by Taub (1965) and Christ (1969) have investigated the effects of differential payoff on the recall of visual symbols. Stimuli used in both of these studies consisted of briefly exposed slides containing randomly selected and positioned letters of the alphabet. Differential payoff was obtained by assigning, to each half of the alphabet, a different value defined by the number of points that could be received for correctly recalling letters within that alphabet half. Separate groups of $S s$ were run, using different ratios of values; the higher-value letters were worth $2,4,8$, or 16 points and the lower-value letters were always worth 1 point. The results of both studies showed that the accuracy of recall was always greater for the higher-value letters than for the letters of lesser value. Furthermore, Taub found that increases in the ratio of high to low value resulted in a decrease in the correct recall of low-value letters, but in essentially constant performance for the higher-value letters.

Since the stimulus displays used in these two studies were exposed for only brief durations and since the letters on each display were randomly positioned without reference to value, both Christ and Taub concluded that the selective nature of the reports was not due to perceptual selectivity but, rather, was due to the selective retrieval of the information stored in short-term memory (STM) during input. However, since $S s$ in these experiments always knew which letters were of higher value before the exposure of a display, these results could be accounted for equally well by reference to a selective mechanism operating during perception (cf. Brown, 1960). Specifically, differential reporting could have resulted from the selective storage and rehearsal of stimulus information as it was perceived. The failure to employ a control to eliminate the possibility of selectivity during perception and storage is a major criticism of the selective-recall interpretation (Egeth, 1967).

The major purpose of the studies reported here was to investigate further the locus of selectivity in tasks employing differential payoff. It was assumed that selectivity could occur either during the perception and concurrent storage of stimulus information (selective retention) or during the retrieval of information stored in STM (selective recall). A partial distinction between the effects of these two loci of selectivity could be made if the stimulus subset designated as being of higher value was allowed to vary from trial to trial and different groups of Ss were instructed either immediately before or immediately after the exposure of each display as to which stimulus subset was of higher value. ${ }^{4}$ The before-exposure instruction (Before) condition should extend and support the earlier findings of Taub and Christ, but, like the conditions employed by those investigators, it would not allow for any distinction between selective retention and selective recall. The after-exposure instruction (After) condition would, however, serve to distinguish partially between the two loci of selectivity since any differentiation between the reports of high- and low-value letters in this condition could only be attributed to selective recall.

\section{Method}

\section{EXPERIMENT 1}

Apparatus. Stimulus displays were projections of 60 negative slides containing eight randomly selected and positioned letters as described by Christ (1969). These slides were constructed using just the first and the last 10 letters of the alphabet, i.e., $A$ through $J$ and $Q$ through $Z$. This procedure provided two subsets of letters, either of which could be designated as the higher-value category for a given slide. There were four A-J letters and four Q-Z letters per slide, and each letter occurred equally of ten over all 60 slides.
Slide exposure time $(1.0 \mathrm{sec})$ was regulated by a solenoid-operated shutter mounted immediately in front of the lens of a Kodak Carousel projector. The changing of slides, exposure time, and interslide interval were controlled by automatic electronic timing equipment. The $S s$ sat in student-type armchairs in groups of 10 so that the projection screen was approximately $15 \mathrm{ft}$ from their eyes; the projection area was $64 \times 44 \mathrm{in}$. and the letters were $1.5 \mathrm{in}$. high. The experimental room was in semi-darkness but light enough for Ss to report their recall from each slide on separate $3 \times 5$ in. cards. These cards were held together by a large ring and each card was specially coded to correspond to a particular slide. On the top of each card for the $S s$ in the differential-value groups was printed "A-J" or "Q-Z," indicating which alphabet subset on the corresponding slide was of higher value. The two alphabet subsets were designated as high valued equally often and the same random order of A-J and Q-Z instructions were given to all experimental groups. Subjects in the control groups, for which the two alphabet subsets were of equal value, had an irrelevant cue, "A-Z," printed on the top of each card.

Subjects. Eighty male volunteers from introductory psychology classes at Kansas State University served as $S$ s in this experiment. These Ss were randomly assigned to eight groups of $10 \mathrm{Ss}$ each. The Ss within each group were run together and competed among themselves for monetary prizes of $\$ 5.00$ and $\$ 3.00$, awarded to the two Ss who accumulated the most points given for correct recall.

Procedure. The experiment was a mixed design, a 2 by 3 factorial with repeated measures at two levels of an additional factor. Value was the within-S factor and referred to the high- and low-value categories of letters designated for each slide independently. The between-S factors were value ratio and instruction time. The value ratios were $2: 1,4: 1$, and $8: 1$, where the first digit referred to the number of points awarded for correctly recalling a high-value letter and the second digit to the number of points awarded for correctly recalling a low-value letter. Two equal-value (1:1) groups were also run to evaluate the effects of instruction time and the initial biases to the letter categories that were, for the 
Table 1

Mean Number of High- and Low-Value Correct Reports for Each Group in Experiment 1

\begin{tabular}{|c|c|c|c|c|c|c|c|c|}
\hline & \multicolumn{8}{|c|}{ Groups } \\
\hline & \multicolumn{4}{|c|}{ Before } & \multicolumn{4}{|c|}{ After } \\
\hline & $1: 1$ & $2: 1$ & $4: 1$ & $8: 1$ & $1: 1$ & $2: 1$ & $4: 1$ & $8: 1$ \\
\hline \multicolumn{9}{|c|}{ High-Valued } \\
\hline $\mathbf{M}$ & 2.22 & 2.07 & 2.04 & 2.02 & 2.03 & 2.08 & 2.16 & 2.23 \\
\hline SD & .29 & .25 & .19 & .23 & .12 & .23 & .15 & .28 \\
\hline \multicolumn{9}{|c|}{ Low-Valued } \\
\hline $\mathbf{M}$ & 2.24 & 2.03 & 1.75 & 1.94 & 2.06 & 1.98 & 2.04 & 2.20 \\
\hline SD & .27 & .25 & .19 & .19 & .16 & .26 & .15 & .26 \\
\hline
\end{tabular}

experimental groups, designated as high- and low-valued. Instruction time referred to the time of specifying the higher-value subset of the alphabet, before or after the exposure of a slide. Since the higher-value subset of the alphabet was always printed on the top of the report cards for the differential-value conditions, the different times of instruction consisted of telling $S s$ in the respective groups to turn to the report card corresponding to a given slide either before or after the exposure of the slide in question. The $\mathrm{E}$ was positioned behind the Ss so that he could monitor the timing of their card-turning activity.

During the first $10 \mathrm{~min}$ of each experimental session, Ss were given general instructions and some training specific to the particular conditions in which they were to be run. All Ss were told of the two categories of letters, and they were further told how points would be accumulated to determine the awarding of prizes. Twelve training slides were used to provide the Ss with practice in seeing the stimulus display and high-value instruction in card turning and in reporting. The 60 experimental slides were presented in the same random order to all groups of Ss.

The Ss in the Before condition were presented a $0.5-\mathrm{sec}$ warning buzzer, followed by a $1.0-\mathrm{sec}$ instruction interval during which they were to turn to the next card and underline the high-value cue. Immediately after this instruction interval, a slide was presented for $1.0 \mathrm{sec}$. Immediately after the slide was exposed, Ss were given $8 \mathrm{sec}$ to record the letters they could recall from the slide. This reporting period was terminated by the 0.5 -sec buzzer announcing the new value instruction interval.

In the After condition, the exposure of a slide was preceded only by the $0.5-\mathrm{sec}$ warning buzzer. The Ss were told to wait until after the slide exposure to turn to the new card and then to underline the high-value letter cue before beginning their report.

Subjects in the two control groups with 1:1 value ratio were given the same tasks to perform in the same sequence as the corresponding differential-value groups but without the differential-value implications.

\section{Results}

Detailed investigation of the results of this experiment included separate analyses for the mean number of correct and false reports of high- and low-value letters per slide. An additional measure consisted of the percentage of initial reports that were higher-value letters. This latter measure was not sensitive to any of the experimental treatments; over all groups, $52.2 \%$ of the initial reports were of high-value letters but deviations from chance expectations were nonsignificant, as were differences between groups.

The mean and SD of correct high- and low-value reports for each differential-value group are shown in Table 1. For comparison, the corresponding data from the two control groups are included in this table. It may be seen that all differential-value groups correctly reported more high-value letters than low-value letters; this was shown to be true for both the Before, $F(1,27)=20.83, p<.01$, and the After, $F(1,27)=4.75, p<.05$, conditions of instruction. The data in Table 1 also show that increases in value ratio have different effects on high- and low-value reports at the two instruction time conditions. This observation is supported by a Value Ratio by Value interaction that was significant for the Before condition, $F(2,27)=6.64$, $p<.05$, but nonsignificant for the After condition, $F<1.0$. Finally, it can be seen that the differential-value groups in the After condition generally made more correct reports than the corresponding groups in the Before condition; the main effect of instruction time was significant, $F(1,54)=7.07, p<.05$. No other effects were significant for the differential-value groups.

In comparison to the differential-value groups, the control groups showed a reversed tendency for selectivity in that they correctly reported slightly more letters from the lower-value category than from the higher-value category (see Table 1). There was also another reversal from the experimental groups in that the Before controls correctly reported more letters than the After controls. An analysis of variance performed on the data from just the two control groups showed, however, that no effects were significant. An analy sis of all four Before groups (including the control) showed a main effect for value ratio, $F(3,36)=4.04, p<.05$. This suggests that the Before control group made significantly more correct reports than the corresponding differential-value groups. This value-ratio main effect over the four After conditions was nonsignificant.

False reports were relatively infrequent, although, for the differential-value groups, there were consistently more false reports of high-value letters than of low-value letters (.46 and .32 per slide, respectively). An analysis of variance of the false-report data from these six groups showed that the only significant influence was value; for the Before groups, $F(1,27)=18.82, p<.01$, and for the After groups, $F(1,27)=6.89$, $\mathrm{p}<.05$.

The similarity of the value effect for both correct and false reports could be an indication that $S s$ were simply guessing some of the higher-value reports. However, if one were to assume that Ss were guessing and hence reporting some high-value letters independent of the contents of STM, these guesses would affect the false reports far more than the correct reports. In all cases, however, the number of correct reports far exceed the number of false reports. Likewise, if guessing were a major factor for the differences between high- and low-value reports, one would expect more false reports from the differential value groups than from the control groups. In fact, while the Before control group did make more errors (1.64) than the After control group (.88), $F(1,18)=6.40, p<.05$, both control groups tended to make more false reports than the corresponding differential-value groups. Subsequent analyses of all four Before groups and all four After groups showed, however, that the main effect of value ratio on false reports was significant for only the Before conditions, $F(3,36)=6.24, p<.01$.

Finally, if the number of correct reports of high-value letters increased only because the total number of reports (correct recalls plus guesses) increased, there should be a strong positive relationship between correct reports and false reports. However, separate product-moment correlations between correct and false reports, computed for each of the six differential-value groups, range from -.09 to +.84 . Only the latter coefficient differed significantly from zero $(\mathrm{df}=8)$, and the pattern of coefficients across conditions was unsystematic and unrelated to the pattern of correct reports.

These comparisons between the relative frequencies of correct and false reports suggest that the greater accuracy in the differential-value groups for the higher-value letters was not due simply to an increased tendency for Ss in these groups to guess and 
report the letters that were worth more points. Instead, the results suggest that these Ss differentially processed the high- and low-valued letters presented in the displays.

\section{EXPERIMENT 2}

The results of Experiment 1 are quite systematic in showing that selective reporting, which favors the higher-value letters, was found for both instruction time conditions and that the Before groups responded at lower overall levels than either their own control or the After groups. To account for these results, it was assumed that attempts to be selective in the perception and retention of higher-value letters, possible only if differential-value instructions are given before the exposure of a display, tend to reduce the overall levels of information available for subsequent recall. It was also assumed that selective recall, which was possible in both instruction time conditions, was primarily responsible for the differential reporting of high-and low-value letters.

If this interpretation is correct, changes in the exposure time of a slide should affect the extent of the instruction time and value effects. Taub (1965) employed three levels of exposure time in his study, $0.5,1.5$, and $2.5 \mathrm{sec}$, and found that increases in exposure time led to overall better performance with a greater benefit going to performance with the higher-value letters than to performance with the lower-value letters. Taub's results, considered in conjunction with the results of Experiment 1, suggest that an increasing amount of time to scan a display leads to more effective selectivity during the perception and retention of input. That is, for the Before groups, an increased exposure time would lead to an overall increase in the number of letters stored in STM but, due to more effective selectivity, the greatest benefit would occur for the higher-value letters. The increasing scan time cannot, however, differentially affect the high- and low-value inputs to STM for the After groups; it will increase the number of letters stored from both categories equally. Hence, the overall levels of performance for the two different times of instruction should be more equal with a relatively long exposure time than with shorter exposure times. However, with increased exposure time, there should be a greater difference in highand low-value reports for the Before group than for the After group. To test these implications, Experiment 2 was conducted.

\section{Method}

Apparatus. Essentially the same materials were used as in Experiment 1 except that only 24 slides were used and each slide contained four A.J letters and four Q.Z letters.

Table 2

Mean Number of High- and Low-Value Correct Reports for Each Group in Experiment 2

\begin{tabular}{|c|c|c|c|c|c|c|c|c|}
\hline & \multicolumn{8}{|c|}{ Groups } \\
\hline & \multicolumn{4}{|c|}{0.5} & \multicolumn{4}{|c|}{2.5} \\
\hline & \multicolumn{2}{|c|}{ Before } & \multicolumn{2}{|c|}{ After } & \multicolumn{2}{|c|}{ Before } & \multicolumn{2}{|c|}{ After } \\
\hline & $1: 1$ & $4: 1$ & $1: 1$ & $4: 1$ & $1: 1$ & $4: 1$ & $1: 1$ & $4: 1$ \\
\hline \\
\hline $\mathbf{M}$ & 1.68 & 1.72 & 1.66 & 1.68 & 2.61 & 3.03 & 2.54 & 2.57 \\
\hline SD & .30 & .19 & .22 & .20 & .41 & .39 & .22 & .36 \\
\hline \\
\hline $\mathbf{M}$ & 1.71 & 1.62 & 1.71 & 1.47 & 2.61 & 2.23 & 2.54 & 2.35 \\
\hline SD & .30 & .26 & .27 & .30 & .41 & .64 & .25 & .48 \\
\hline
\end{tabular}

Subjects. Sixty-four male volunteers from introductory psychology dasses served as Ss in this experiment. These Ss were randomly assigned to groups of eight Ss each. As in Experiment 1, the Ss within each group were run together and competed among themselves for monetary prizes of $\$ 5.00$ and $\$ 3.00$.

Procedure. The experiment was a mixed design, a 2 by 2 factorial with repeated measures at two levels of an additional factor. Value was the within-S factor and exposure time and instruction time were the between-S factors. Exposure times for the slides were either 0.5 or $2.5 \mathrm{sec}$. The factors of value and instruction time were the same as in Experiment 1. All Ss in the experimental groups were run under a 4:1 value ratio, defined as in Experiment 1. Four additional, equal-value (1:1) groups were run as controls.

The general procedure both for training and running Ss was as in Experiment 1. The total time for seeing and reporting a given slide varied across different levels of exposure time, but the warning buzzer, preparation interval, and reporting interval were as before.

\section{Results}

The results of this experiment were analyzed independently for the mean number of correct and false reports of highand low-value letters per slide and for the percentage of times the initial response was a high-value letter. The latter analy sis showed that only the $2.5 \mathrm{sec}, 4: 1$-Before group gave more first reports of high value than would have been expected by chance. Hence, while the first letter reported by this group was a high-value letter on $72.1 \%$ of the slides, the other seven groups reported high-value letters first on $51.8 \%$ of the slides.

Table 2 shows the mean number and SD of correct high- and low-value reports for each of the differential-value groups and for the four control groups. It can be seen that there were more correct reports given in the 2.5-sec exposure condition than in the 0.5 -sec exposure condition. This was true at the .01 level of significance for the differential-value groups, $F(1,28)=64.99$.
The data in Table 2 also show more highthan low-value correct reports for all four differential-value groups, $F(1,28)=22.70$, $\mathrm{p}<.01$, but generally the differences are greater for the groups with the longer exposure, as shown by the significant Exposure Time by Value interaction, $F(1,28)=6.36, \quad p<.05$. Finally, differential responding tended to be greater for the After condition than the Before with $0.5 \mathrm{sec}$ of exposure, but the reverse is true with the 2.5-sec exposure. This latter effect is supported by a significant Exposure Time by Instruction Time by Value interaction, $F(1,28)=5.81, p<.05$. Separate analyses for each of the four differential-value groups yielded no significant effect for either group at the 0.5 -sec exposure, but value was significant for both the Before and After groups at the $2.5-\mathrm{sec}$ exposure, $\mathrm{F}(1,7)=13.25$ and $9.67, \mathrm{p}<.01$ and .05 , respectively.

The four control groups show the same beneficial effect of increased exposure time, $F(1,28)=82.16, p<.01$, but no effect due to the instruction time or value variables. Comparison between the four pairs of corresponding control and differential-value groups in Table 2 suggests that while performance with the higher-value category of letters remains constant or increases, performance with the lower-value letters always decreases when differential value is introduced. Separate analyses on all four pairs of control and differential-value groups show that this Value Ratio by Value interaction is significant only for the $2.5-\mathrm{sec}$ exposure conditions, $F(1,14)=12.03$, $p<.01$ for the Before groups and $F(1,14)=7.62, \quad p<.05$ for the After groups.

The frequency of false reports was relatively small, but there was a slight tendency for $S s$ in the differential-value groups to make more false reports of high-value letters than of low-value letters (.38 and .30 per slide, respectively). An analysis of variance for these data, however, failed to show any significant effects. As in Experiment 1, analyses of the relative frequencies of correct and false reports suggest that the effects found for 
differential value were not due to differential guessing. If chance responding and guessing were important, one would expect many more false reports than were found. Similarly, if guessing increases as a result of differential values, one would expect more errors for the differential-value groups than for the controls. However, comparisons between the four pairs of corresponding control and experimental groups showed that there was always a decrease in errors going from the equal-value to the differential-value condition. On the average, $S s$ in the control groups made 1.20 false reports per slide, while $S s$ in the differential-value groups made only .34 . Separate analyses on the four pairs of conditions showed that this decrease was significant for only the 0.5-sec After conditions, $F(1,56)=9.80, \quad p<.01$. Finally, the correlations between correct and false reports computed separately for the four experimental groups produced coefficients that ranged from -.34 to +.30 . For all rs, with $6 \mathrm{df}, \mathrm{p}>.05$.

\section{DISCUSSION AND CONCLUSIONS}

The results of these experiments support and extend the generality of those of Taub (1965) and Christ (1969) by showing a bias in reporting differentially valued letters that, for the After condition, could only be explained by reference to selective retrieval from STM. However, at the longer exposures, where Ss could scan the entire slide more than once, the differential effect of value was smaller for the After condition than for the Before condition. These data suggest that, under appropriate conditions, selectivity also occurs during the perception and retention of stimulus materials and that this mode or locus of selectivity enhances the selective recall effects. That is, biasing of reports may be much greater for $S s$ in the Before condition for whom both loci of selectivity operate than for $S s$ in the After condition for whom there is only selective recall. The fact that initial reports of higher-value letters are significantly different from chanœ expectations only for the Before condition and only at the longest exposure tends to support this conclusion.

The data furthermore show that as exposure time increased, the difference between performance with high- and low-value letters increased for both instruction time conditions but more for the Before condition than for the After condition. The increased selectivity with increased exposure time is in agreement with Taub's (1965) results. That this effect is greater for the Before condition than for the After condition suggests that selectivity during perception and retention is more effective at the longer exposures than at the shorter exposures.

The effects of value over levels of differential-value ratio reported by $T$ aub and as tested in Experiment 1 were not found. Hence, while Taub found essentially constant performance with high-value letters and decreasing performance with low-value letters as the ratio of differential values increased, the differential-value groups in Experiment 1 show unsystematic effects resulting from these variables. There was, however, a general tendency for the total frequency of both correct and false reports to decrease for the differential-value groups relative to their respective control groups. Hence, in 7 of the 10 possible comparisons, correct responses decrease, and in 9 of 10 comparisons, false responses decrease in the differential-value groups relative to their respective control groups. These latter results suggest that $S$ s in the differential-value groups are attempting to minimize errors rather than maximize correct reports.

\section{REFERENCES}

BROWN, J. Evidence for a selective process during perception of tachistoscopically presented stimuli. Journal of Experimental Psychology, $1960,59,176-181$.

CHRST, R. E. Effects of payoff and probability on recall of multisymbol displays. Joumal of General Psychology, 1969, 80, 81-92.

EGETH, H. Selective attention. Psychological Bulletin, 1967, 67, 41-57.

EGETH, H., \& SMITH,E. E. Perceptual selectivity in a visual recognition task. Journal of Experimental Psychology, 1967, 74, 543-549.

TAUB, H. A. Effects of differential value on recall of visual symbols. Journal of Experimental Psychology, 1965, 69, 135-143.

\section{NOTES}

1. This research was supported by a faculty research grant, $F 314$, from the Bureau of General Research, Kansas State University. The first experiment reported is based upon a thesis submitted by the first author to Kansas State University in partial fulfillment of the requirements for the degree of Master of Science. Portions of this paper were presented at the 1967 Convention of the Midwestern Psychological Association.

2. Now at the University of Melbourne.

3. Address: Department of Psychology, Anderson Hall, Kansas State University, Manhattan, Kansas 66502.

4. The methods employed here really provide for a Before and After vs an After comparison, in keeping with the arguments raised by Egeth and Smith (1967). The former will, for the sake of brevity, be referred to as the before-exposure instruction condition.

(Accepted for publication April 28, 1969.) 